17

\title{
О термической устойчивости некоторых квазифуллеренов
}

\author{
(c) А.И. Подливаев ${ }^{1,2}$, Л.А. Опенов ${ }^{1}$ \\ ${ }^{1}$ Национальный исследовательский ядерный университет „МИФИ“, \\ Москва, Россия \\ ${ }^{2}$ Научно-исследовательский институт проблем развития \\ научно-образовательного потенциала молодежи, \\ Москва, Россия \\ E-mail: AIPodlivayev@mephi.ru
}

(Поступила в Редакцию 28 сентября 2018 г.)

Методом молекулярной динамики изучена термическая устойчивость недавно предсказанных квазифуллеренов $\mathrm{C}_{20}, \mathrm{C}_{42}, \mathrm{C}_{48}$ и $\mathrm{C}_{60}$. Определены каналы их распада и температурные зависимости времени жизни. Найдены значения энергий активации и частотных факторов в законе Аррениуса. Обнаружены новые изомеры.

Работа поддержана грантом РФФИ № 18-02-00278-а и выполнена при поддержке Министерства образования и науки РФ в рамках Программы повышения конкурентоспособности НИЯУ МИФИ.

DOI: 10.21883/FTT.2019.03.47258.264

\section{1. Введение}

Двумерные (графен [1]) и одномерные (нанотрубки [2], карбин [3]) углеродные материалы представляют как фундаментальный, так и практический интерес. Это же относится к нульмерным структурам - фуллеренам [4] и другим атомным кластерам [5-8].

Существуют различные схемы классификации углеродных фаз вообще [9] и фуллеренов в частности [10,11]. Классические фуллерены представляют собой замкнутые клетки с шестиугольными и пятиугольными гранями, в которых каждый атом углерода образует ковалентные связи с тремя своими соседями ( $s p^{2}$-гибридизация электронных орбиталей). В неклассических фуллеренах и квазифуллеренах могут также присутствовать четырехзвенные, семизвенные, восьмизвенные и др. углеродные кольца [12-14]. В отдельную группу можно выделить карбинофуллерены с карбиновыми цепочками [15], где часть атомов имеет двух соседей и находится в состоянии $s p$-гибридизации.

Для приготовления квазифуллеренов используют как известные, так и новые методы [16], но экспериментальная информация об их физико-химических свойствах остается весьма скудной. В этой ситуации особое значение приобретает компьютерное моделирование. Недавно на основании численных расчетов было предсказано несколько новых карбинофуллеренов и квазифуллеренов [9,17]. В работе [17] методами теории функционала плотности детально изучены структура, электронные и химические свойства квазифуллеренов $\mathrm{C}_{n}$ с $n=20,42$, 48 и 60 (рис. 1).

Важной характеристикой атомных кластеров является их термическая устойчивость - способность сохранять свою структуру при нагревании. Как показано в работе [17], во всех изученных авторами квазифуллеренах при температуре $T=800-1000 \mathrm{~K}$ разрыва межатомных связей не происходит, по крайней мере за время $t=1 \mathrm{ps}$. Целью настоящей работы является компьютерное моделирование временно́й эволюции квазифуллеренов $\mathrm{C}_{n} \mathrm{c}$ $n=20,42,48$ и 60 в течение гораздо более длительного времени, вплоть до момента распада. Это позволяет определить возможные каналы распада кластеров и температурные зависимости их времен жизни, а также найти новые изомеры, в том числе с более низкой энергией.

\section{2. Методы расчета}

Для моделирования динамики квазифуллеренов мы использовали микроканонический NVE-ансамбль [18], в котором полная энергия кластера $E$ (сумма потенциальной и кинетической энергии) остается постоянной в процессе его эволюции. Роль температуры при этом играет так называемая „микроканоническая“ (или „динамическая“) температура $T_{m}$, являющаяся мерой кинетической энергии относительного движения атомов в кластере, который как целое покоится и не вращается (то есть мерой энергии возбуждения кластера). Она вычисляется по формуле $[19,20]\left\langle E_{\mathrm{kin}}\right\rangle=\frac{1}{2} k_{\mathrm{B}} T_{m}(3 n-6)$, где $\left\langle E_{\mathrm{kin}}\right\rangle-$ усредненная по времени кинетическая энергия кластера, $n$ - число атомов в нем, $k_{\mathrm{B}}$ - постоянная Больцмана.

Такой подход лежит в основе теории ограниченного теплового резервуара (finite heat bath theory) [21,22], согласно которой сам кластер играет роль резервуара для степени свободы вдоль координаты реакции, приводящей к его распаду. Строго говоря, он применим либо к теплоизолированным кластерам, либо в ситуациях, когда распад кластера после его формирования происходит настолько быстро, что тепловое равновесие с окружением не успевает установиться. В других случаях этот 

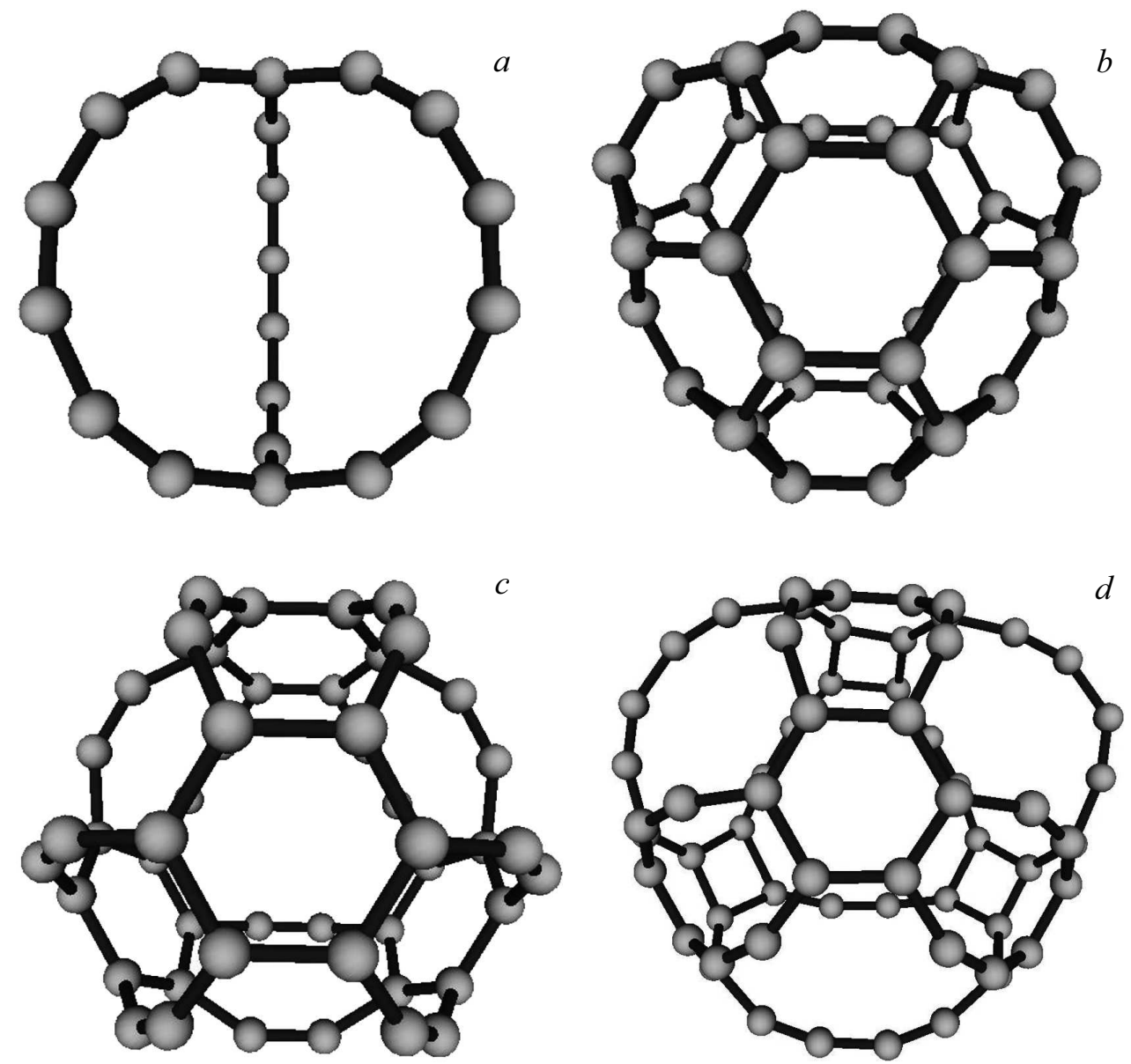

Рис. 1. Квазифуллерены $\mathrm{C}_{20}(a), \mathrm{C}_{42}(b), \mathrm{C}_{48}(c), \mathrm{C}_{60}(d)$.

подход дает качественную информацию о поведении метастабильной атомной системы при возбуждении.

Мы проводили моделирование следующим образом. В исходный момент времени всем атомам сообщались начальные скорости так, чтобы импульс и момент импульса кластера как целого были равны нулю. Затем по формуле Хельмана-Фейнмана вычислялись действующие на атомы силы. Для этой цели использовалась неортогональная модель сильной связи [23], которая хотя и уступает по точности первопринципным методам, но требует значительно меньше компьютерных ресурсов и потому позволяет проследить за эволюцией атомных кластеров в течение гораздо более длительного времени. Ранее эта модель продемонстрировала свою применимость к численному моделированию различных углеродных нанотруктур (см. работы [24-26] и ссылки в них). После нахождения межатомных сил уравнения движения Ньютона решались численно методом скоростей Верле с шагом по времени $0.3 \mathrm{fs}$. Для визуализации процесса эволюции кластера мы периодически записывали координаты всех атомов, а затем представляли полученные результаты в виде компьютерной анимации.

Для анализа температурной зависимости времени $\tau$ протекания того или иного термоактивированного про- цесса (распада кластера, его изомеризации и пр.) мы используем формулу Аррениуса, модифицированную с учетом конечных размеров теплового резервуара $[21,22]$,

$$
\tau^{-1}\left(T_{m}\right)=A \cdot \exp \left(-\frac{E_{a}}{k_{\mathrm{B}} T^{*}}\right)
$$

где $A-$ частотный фактор, $E_{a}-$ энергия активации, $T^{*}=T_{m}-E_{a} / 2 C, C=(3 n-6) k_{\mathrm{B}}-$ микроканоническая теплоемкость кластера. Величину $A$ мы рассчитывали по формуле Виньярда [27] после определения спектров собственных колебаний в соответствующих стационарных точках поверхности потенциальной энергии (potential energy surface, PES).

\section{3. Результаты и их обсуждение}

\section{1. Квазифуллерен $\mathbf{C}_{20}$}

Изображенный на рис. $1, a$ кластер $\mathrm{C}_{20}$ авторы работы [17] называют квазифуллереном, хотя на наш взгляд правильнее было бы говорить о нем как о карбинофуллерене [15]. Этот кластер образован тремя 8 атомными карбиновыми цепочками с двумя общими атомами 
(вершинами). По своей форме он напоминает мяч для игры в регби. Для его энергии связи, определяемой как разность между полной энергией кластера и энергией составляющих изолированных атомов углерода, мы нашли $E_{b}=-6.68 \mathrm{eV} /$ atom.

Моделирование динамики кластера проводилось при температурах $T_{m}=1600-3600 \mathrm{~K}$. В процессе эволюции кластера мы несколько раз наблюдали, как после разрыва ближайшей к одной из вершин связи С-C и последующего формирования новой связи образуется изомер, состоящий из 7-атомной, 8-атомной и 9-атомной карбиновых цепочек (рис. 2, $a$ ). Этот изомер имеет более высокую энергию связи $E_{b}=-6.62 \mathrm{eV} /$ atom, то есть является энергетически менее выгодным. Анализ PES дает $U=2.6 \mathrm{eV}$ для высоты барьера, препятствующего его образованию, и $A=4.0 \cdot 10^{14} \mathrm{~s}^{-1}$ для частотного фактора процесса изомеризации. Полагая $E_{a}=U$ в формуле (1) и принимая во внимание наличие 12 эквивалентных путей изомеризации кластера $\mathrm{C}_{20}$, оценим характерное время изомеризации $\sim 10 \mathrm{~ns}$ при $T_{m}=2000 \mathrm{~K}$ и $\sim 5 \min$ при $T_{m}=1000 \mathrm{~K}$.

Если после разрыва одной из связей $\mathrm{C}-\mathrm{C}$ новая связь не формируется, то имеет место необратимый распад кластера. В результате образуется атомная конфигурация, имеющая форму теннисной ракетки (рис. 2,b). Ее энергия связи $E_{b}=-6.66 \mathrm{eV} /$ atom. На рис. 3 представлены расчетные значения времени жизни $\tau$ карбинофуллерена $\mathrm{C}_{20}$ до момента его распада при различных температурах. Видно, что температурная зависимость $\tau$ подчиняется закону Аррениуса. Статистическая обработка данных „численного экспе-

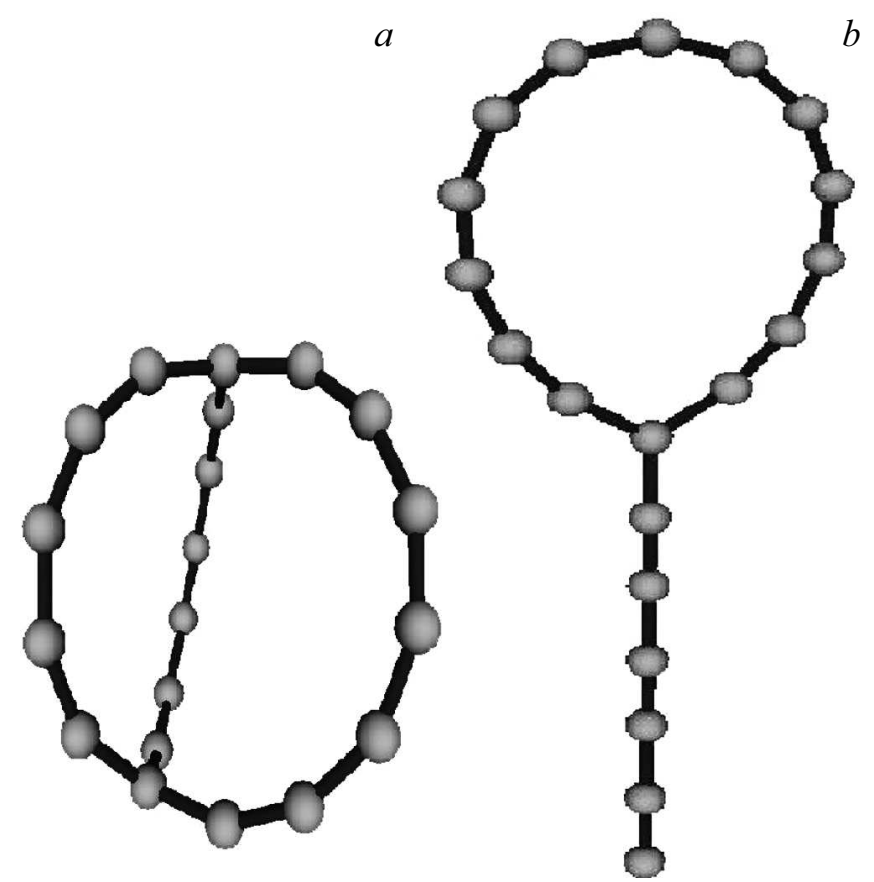

Рис. 2. Изомер $(a)$ и конечный продукт распада $(b)$ карбинофуллерена $\mathrm{C}_{20}$.

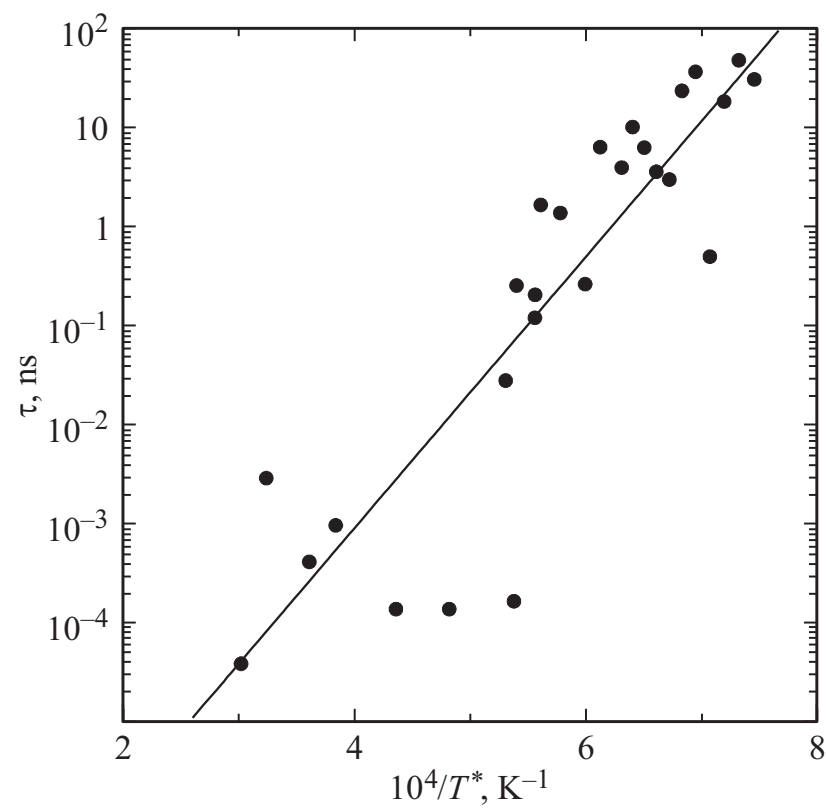

Рис. 3. Зависимость времени жизни $\tau$ карбинофуллерена $\mathrm{C}_{20}$ от обратной температуры $T^{*}$ (см. текст). Точки - результаты численного счета. Сплошная линия - линейная аппроксимация методом наименьших квадратов.

римента" дает следующие значения энергии активации и частотного фактора распада: $E_{a}=2.72 \pm 0.31 \mathrm{eV}$, $A=(3.7 \pm 1.6) \cdot 10^{17} \mathrm{~s}^{-1}$. Для времени распада кластера по формуле (1) получим $\tau \sim 0.5 \mu \mathrm{s}$ при $T_{m}=1500 \mathrm{~K}$, $\tau \sim 1 \min$ при $T_{m}=1000 \mathrm{~K}$ и т.д.

\section{2. Квазифуллерены $\mathrm{C}_{42}$ и $\mathrm{C}_{48}$}

В структуре квазифуллеренов $\mathrm{C}_{42}$ (рис. $\left.1, b\right)$ и $\mathrm{C}_{48}$ (рис. $1, c)$ имеется различное количество 6-звенных и 8-звенных углеродных колец (детали см. в работе [17]). Кроме того, в квазифуллерене $\mathrm{C}_{42}$ имеется несколько 4-звенных колец, форма которых близка к прямоугольной. Оба кластера содержат также большие 12-звенные кольца. Для энергий связи этих кластеров мы получили $E_{b}=-6.54 \mathrm{eV} /$ atom и $-6.75 \mathrm{eV} /$ atom соответственно.

Мы моделировали временну́ю эволюцию квазифуллеренов $\mathrm{C}_{42}$ и $\mathrm{C}_{48}$ при температурах $T_{m}=900-1900 \mathrm{~K}$ и $T_{m}=1700-2500 \mathrm{~K}$ соответственно. Мы обнаружили, что распад кластера $\mathrm{C}_{42}$ всегда начинается с разрыва связи С-C в одном из 4-звенных колец. После разрыва всех связей в 4-звенных кольцах образуется изомер, изображенный на рис. 4, $a$. Его энергия связи $E_{b}=-6.67 \mathrm{eV} /$ atom меньше, чем у исходного квазифуллерена. Так как после разрыва первой связи распад становится необратимым, то время ее разрыва мы принимали за время жизни квазифуллерена $\tau$. Расчетная температурная зависимость $\tau$ приведена на рис. 5, a. Она описывается законом Аррениуса с параметрами $E_{a}=1.19 \pm 0.11 \mathrm{eV}, A=(6.6 \pm 1.7) \cdot 10^{14} \mathrm{~s}^{-1}$. 

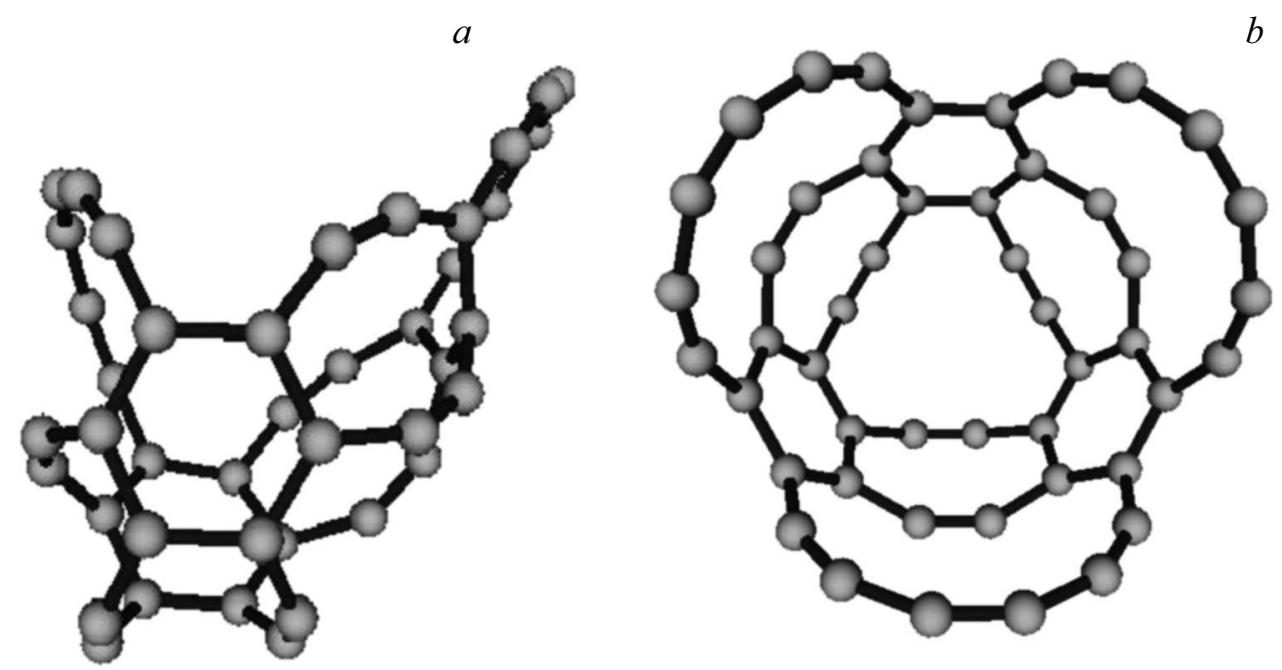

Pис. 4. Изомеры, образующиеся при распаде квазифуллеренов $\mathrm{C}_{42}(a)$ и $\mathrm{C}_{48}(b)$.
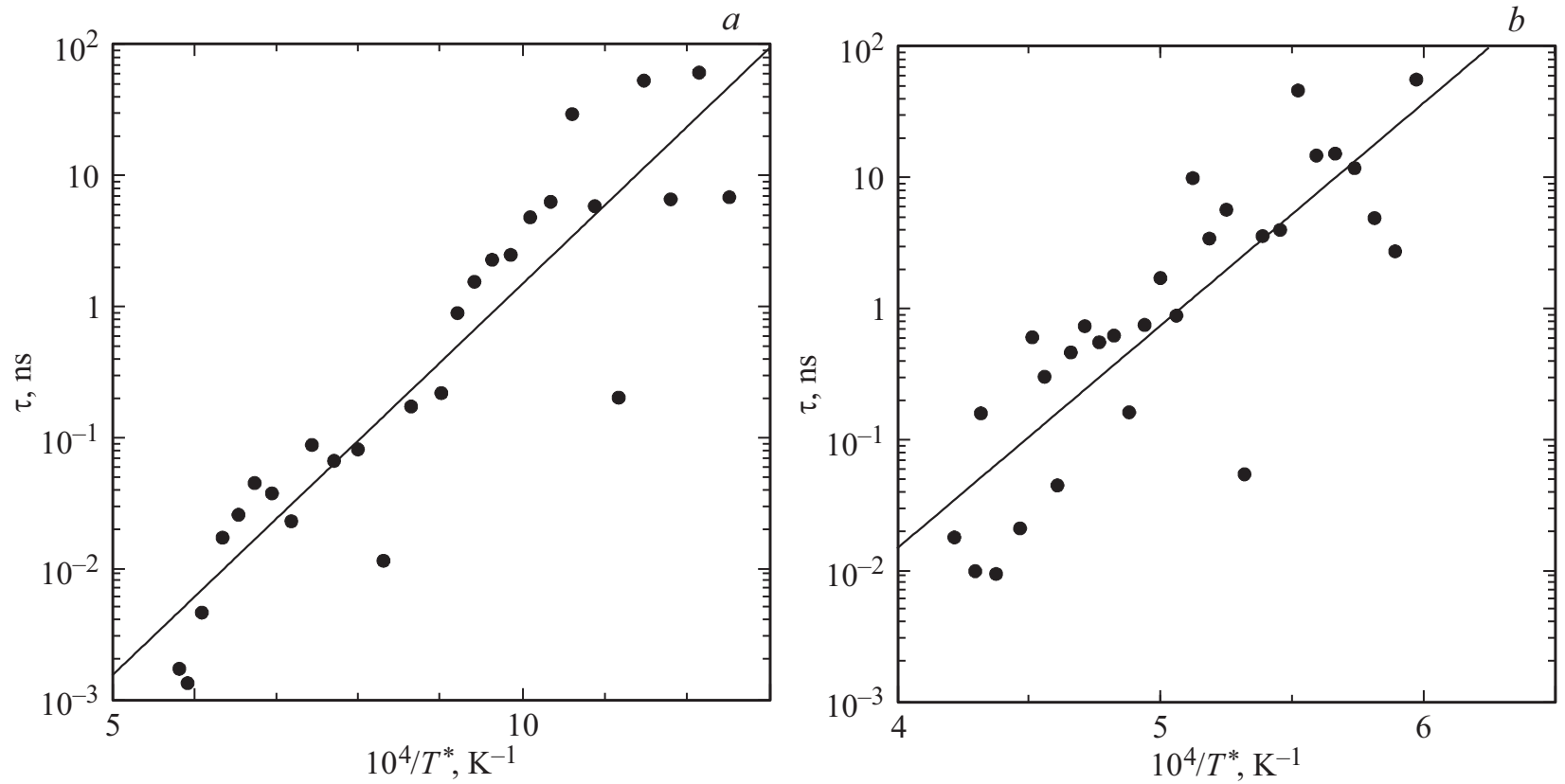

Рис. 5. То же, что на рис. 3 , для квазифуллеренов $\mathrm{C}_{42}(a)$ и $\mathrm{C}_{48}(b)$.

Например, при $T_{m}=1000$ и $500 \mathrm{~K}$ из формулы (1) найдем $\tau \sim 3 \mathrm{~ns}$ и $\tau \sim 50 \mathrm{~ms}$ и соответственно.

Распад квазифуллерена $\mathrm{C}_{48}$ чаще всего инициируется разрывом одной из связей $\mathrm{C}-\mathrm{C}$, которые являются общими для 6-звенного и 8-звенного колец, реже разрывом связи, общей для 8-звенного и 12-звенного колец. После разрыва первой такой связи распад кластера становится необратимым, поэтому время ее разрыва мы принимали за время жизни $\tau$ этого квазифуллерена. Вслед за разрывом еще нескольких связей образуется изображенный на рис. $4, b$ изомер с более низкой энергией связи $E_{b}=-6.77 \mathrm{eV} /$ atom.

Результаты расчета $\tau$ при различных температуpax приведены на рис. 5, $b$. Температурная зависи- мость $\tau$ описывается формулой (1) с параметрами $E_{a}=3.37 \pm 0.45 \mathrm{eV}$ и $A=(4.3 \pm 1.1) \cdot 10^{17} \mathrm{~s}^{-1}$. В частности, при $T_{m}=1500$ и $1000 \mathrm{~K}$ имеем $\tau \sim 10 \mu \mathrm{s}$ и $\tau \sim 1 \mathrm{~min}$ соответственно.

\section{4. Квазифуллерен $\mathrm{C}_{60}$}

Отличительной особенностью квазифуллерена $\mathrm{C}_{60}$ (рис. $1, d$ ) является то, что наряду с 6-звенными и 8-звенными углеродными кольцами в его структуре имеются также три больших 14-звенных кольца и три маленьких квадратных кольца. Энергия связи кластера составляет $E_{b}=-6.73 \mathrm{eV} /$ atom. 


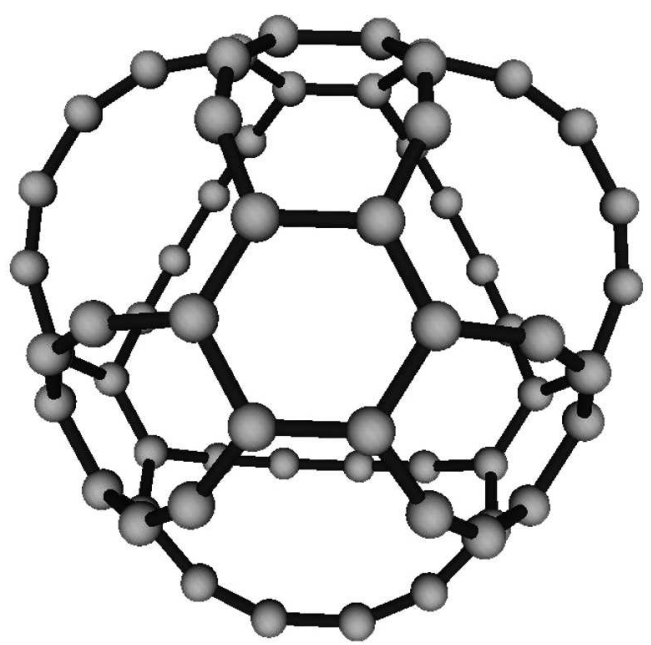

Рис. 6. Изомер, образующийся при распаде квазифуллерена $\mathrm{C}_{60}$.

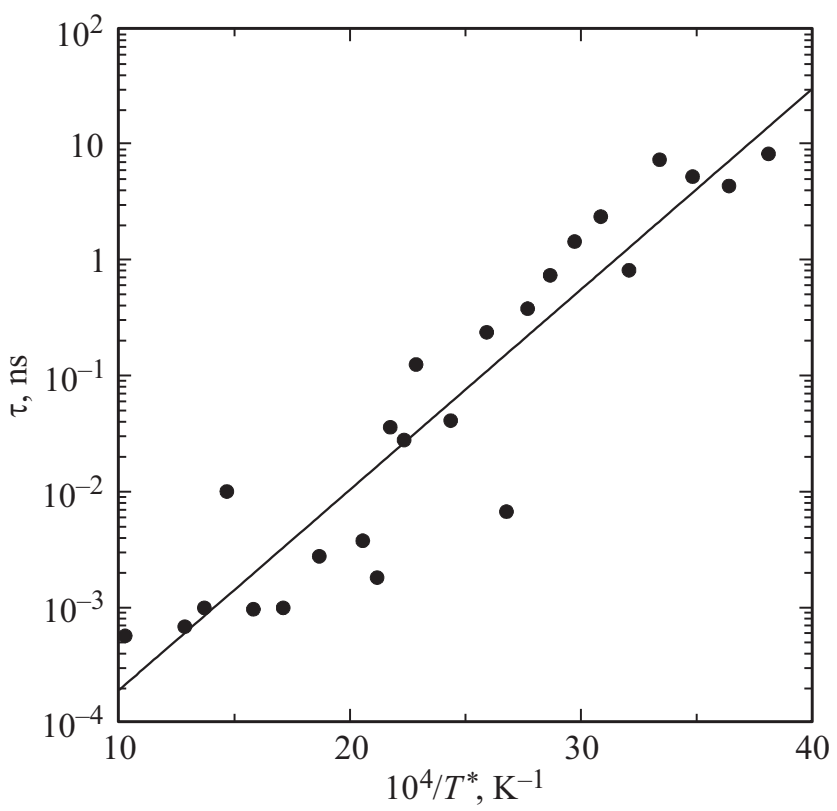

Pис. 7. То же, что на рис. 3 , для квазифуллерена $\mathrm{C}_{60}$.

Динамику квазифуллерена $\mathrm{C}_{60}$ мы моделировали при температурах $T_{m}=300-2000 \mathrm{~K}$. За время его жизни $\tau$ мы принимали время разрыва связи $\mathrm{C}-\mathrm{C}$ в одном из квадратных колец, так как вслед за этим следует быстрый разрыв связей в других квадратных кольцах, формируется большое 18-звенное кольцо и образуется изомер с более низкой энергией связи $E_{b}=-6.81 \mathrm{eV} /$ atom (рис. 6). Результаты расчета $\tau$ приведены на рис. 7. Температурная зависимость $\tau$ описывается формулой (1) с параметрами $E_{a}=0.33 \pm 0.03 \mathrm{eV}$ и $A=(2.1 \pm 0.5) \cdot 10^{14} \mathrm{~s}^{-1}$. Малая величина энергии активации является признаком низкой термической устойчивости. Действительно, $\tau \sim 1$ ps при $T_{m}=1000 \mathrm{~K}$ и $\tau \sim 3 \mathrm{~ns}$ при $T_{m}=300 \mathrm{~K}$. Охлаждение до $T_{m}=100 \mathrm{~K}$ приводит к увеличению $\tau$ до $\sim 6 \mathrm{~h}$.

Изомер $\mathrm{C}_{60}$ (рис. 6) значительно более устойчив к нагреванию. Мы изучили его временну́ю эволюцию в диапазоне температур $T_{m}=2000-3000 \mathrm{~K}$. Распад этого изомера начинается с практически одновременного разрыва трех связей С-C в одном из 6-звенных колец, после чего кластер быстро теряет свою сфероидальную форму (рис. 8). Температурная зависимость времени жизни $\tau$ приведена на рис. 9. Она подчиняется закону Аррениса с параметрами $E_{a}=3.82 \pm 0.39 \mathrm{eV}$ и $A=(9.8 \pm 2.6) \cdot 10^{18} \mathrm{~s}^{-1}$. По формуле (1) получим, в частности, $\tau \sim 10 \mu \mathrm{s}$ при $T_{m}=1500 \mathrm{~K}$ и $\tau \sim 20 \min$ при $T_{m}=1000 \mathrm{~K}$. Таким образом, изомер $\mathrm{C}_{60}$ оказывается

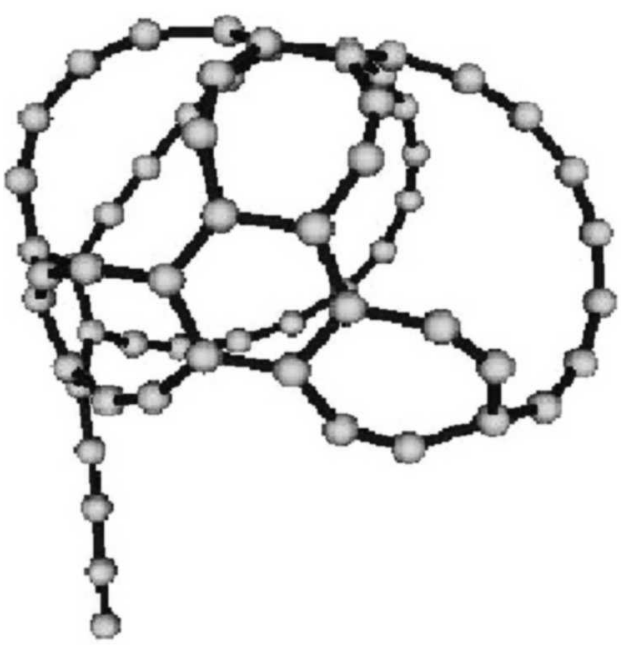

Рис. 8. Атомная конфигурация, образующаяся после распада изомера квазифуллерена $\mathrm{C}_{60}$.

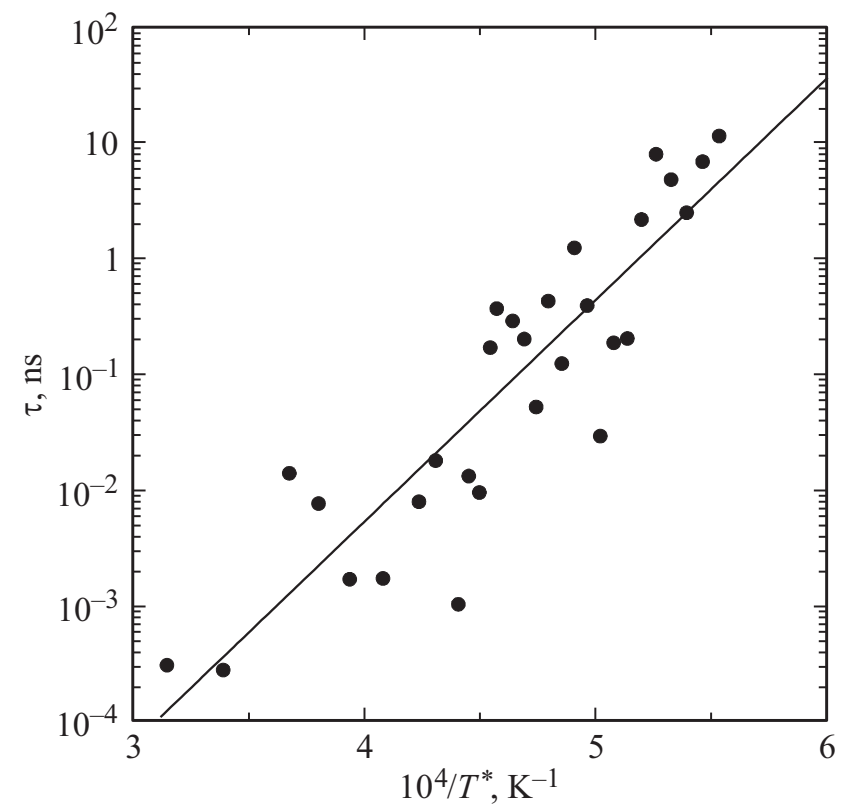

Рис. 9. То же, что на рис. 3 , для изомера квазифуллерена $\mathrm{C}_{60}$. 
гораздо устойчивее исходного квазфуллерена не только термодинамически, но и термически.

\section{5. Заключение и выводы}

В настоящей работе термическая устойчивость карбинофуллерена $\mathrm{C}_{20}$ и квазифуллеренов $\mathrm{C}_{42}, \mathrm{C}_{48}, \mathrm{C}_{60}$ изучена путем компьютерного моделирования на наносекундной временно́й шкале в широком диапазоне температур. Определены температурные зависимости времен жизни $\tau$ этих кластеров до момента их распада. Установлено, что карбинофуллерен $\mathrm{C}_{20}$ и квазифуллерен $\mathrm{C}_{48}$ достаточно устойчивы к нагреву до высоких температур: для них $\tau \sim 1-10 \min$ при $T=1000 \mathrm{~K}$. Из всех рассмотренных нами кластеров наинизшую термическую устойчивость имеет квазифуллерен $\mathrm{C}_{60}$ : для него $\tau \sim 1 \mathrm{ps}$ при $T=1000 \mathrm{~K}$.

После распада квазифуллерены $\mathrm{C}_{42}, \mathrm{C}_{48}$ и $\mathrm{C}_{60}$ переходят в изомеры с более низкой энергией. При этом термическая устойчивость изомера $\mathrm{C}_{60}$ оказывается значительно выше, чем у исходного квазифуллерена: Например, при $T=1000 \mathrm{~K}$ время его жизни составляет около $20 \mathrm{~min}$.

Мы надеемся, что полученные результаты будут полезны при разработке методов изготовления новых карбинофуллеренов и квазифуллеренов и/или их изомеров.

Мы признательны J. Muñiz за подробную информацию об атомной структуре изученных в настоящей работе квазифуллеренов.

\section{Список литературы}

[1] K.S. Novoselov, A.K. Geim, S.V. Morozov, D. Jiang, Y. Zhang, S.V. Dubonos, I.V. Grigorieva, A.A. Firsov. Science 306, 666 (2004).

[2] S. Iijima. Nature 354, 56 (1991).

[3] В.И. Касаточкин, А.М. Сладков, Ю.П. Кудрявцев, Н.М. Попов, В.В. Коршак. ДАН СССР 177, 358 (1967).

[4] H.W. Kroto, J.R. Heath, S.C. O'Brien, R.F. Curl, R.E. Smalley. Nature 318, 162 (1985).

[5] D. Tománek, M.A. Schluter. Phys. Rev. Lett. 67, 2331 (1991).

[6] C.H. Xu, C.Z. Wang, C.T. Chan, K.M. Ho. Phys. Rev. B 47, 9878 (1993).

[7] R.O. Jones, G. Seifert. Phys. Rev. Lett. 79, 443 (1997).

[8] L.A. Openov, V.F. Elesin. Письма в ЖЭТФ 68, 695 (1998).

[9] Е.А. Беленков, В.А. Грешняков. ФТТ 55, 1640 (2013).

[10] E.W. Godly, R. Taylor. Fuller. Sci. Technology 5, 1667 (1997).

[11] V. Georgakilas, J.A. Perman, J. Tucek, R. Zbori. Chem. Rev. 115, 4744 (2015).

[12] L.-H. Gan, J.-Q. Zhao, F. Pan. J. Mol. Struct.: Theochem 953, 24 (2010).

[13] Z. Slanina, X. Zhao, Ōsawa. Chem. Phys. Lett. 290, 311 (1998).

[14] B.I. Dunlap, R. Taylor. J. Phys. Chem. 98, 11018 (1994).

[15] Е.А. Беленков, И.В. Шахова. ФТТ 53, 2265 (2011).

[16] А.И. Харламов, Г.А. Харламова, М.Э. Бондаренко. ЖПХ 86, 1198 (2013).
[17] C.A. Celaya, J. Muñiz, L.E. Sansores. Comp. Theor. Chem. 1117, 20 (2017).

[18] E.M. Pearson, T. Halicioglu, W.A. Tiller. Phys. Rev. A 32, 3030 (1985).

[19] C. Xu, G.E. Scuseria. Phys. Rev. Lett. 72, 669 (1994).

[20] J. Jellinek, A. Goldberg. J. Chem. Phys. 113, 2570 (2000).

[21] C.E. Klots. Z. Phys. D 20, 105 (1991).

[22] J.V. Andersen, E. Bonderup, K. Hansen. J. Chem. Phys. 114, 6518 (2001).

[23] M.M. Maslov, A.I. Podlivaev, K.P. Katin. Mol. Simul. 42, 305 (2016).

[24] А.И. Подливаев, Л.А. Опенов. ФТТ 60, 160 (2018).

[25] K.S. Grishakov, K.P. Katin, M.M. Maslov. Adv. Phys. Chem. 2016, 1862959 (2016).

[26] Л.А. Опенов, А.И. Подливаев. ФТТ 59, 1240 (2017).

[27] G.V. Vineyard. J. Phys. Chem. Solids 3, 121 (1957).

Редактор К.В. Емцев 